\title{
Polarized Emission from Conjugated Polymer Chains Aligned by Epitaxial Growth during Off-Center Spin-Coating
}

\author{
Takuya Anzai, ${ }^{1}$ William Porzio, ${ }^{2}$ and Varun Vohra ${ }^{1}$ \\ ${ }^{1}$ Department of Engineering Science, University of Electro-Communications, 1-5-1 Chofugaoka, Chofu 182-8585, Japan \\ ${ }^{2}$ Istituto per lo Studio delle Macromolecole (ISMAC-CNR), Via Corti 12, 20133 Milano, Italy \\ Correspondence should be addressed to Varun Vohra; varun.vohra@uec.ac.jp
}

Received 25 July 2017; Accepted 13 September 2017; Published 17 October 2017

Academic Editor: Yves Grohens

Copyright (c) 2017 Takuya Anzai et al. This is an open access article distributed under the Creative Commons Attribution License, which permits unrestricted use, distribution, and reproduction in any medium, provided the original work is properly cited.

\begin{abstract}
Due to their macromolecular nature, conjugated polymers can be relatively easily aligned by applying a variety of processes resulting in either elongation or ordering of their conjugated backbones. Processes that induce chain alignment include electrospinning, mechanical rubbing, epitaxial growth, and nanoconfinement and unidirectional deposition techniques such as off-center spincoating. In this study, we compare these deposition techniques by applying them to a green-emitting conjugated polymer material that exhibits liquid crystalline phase behavior. Our study reveals that while methods such as electrospinning and mechanical rubbing can be useful to locally generate polymer chain alignment, the combination of epitaxial growth using 1,3,5-trichlorobenzene as crystallizing agent with off-center spin-coating results in the formation of anisotropic nanofiber-like structures with enhanced crystallinity degree and polarized light-emission properties. The unidirectional epitaxial growth was also applied to a red-emitting polymer that exhibits polarization ratios up to 4.1. Our results emphasize that this simple solution formulation and process can be used for the fabrication of polarized thin films of a variety of conjugated polymers with potential applications in the advanced display technologies or analytical equipment fields.
\end{abstract}

\section{Introduction}

Conjugated light-emitting polymers have been increasingly studied since the pioneering work from Burroughes et al. in which they were used in light-emitting devices exhibiting high luminance and the potential for large-scale device fabrication using simple solution processes [1]. In particular, these materials have the potential to be relatively easily oriented as the polymer chains can be mechanically aligned using a variety of processes [2]. Such processes include the formation of nano- or microfibers by electrospinning $[3,4]$, mechanical rubbing $[5,6]$, unidirectional deposition [7], and epitaxial growth $[8,9]$. The resulting films often display enhanced optical or charge transport properties and thin films with aligned conjugated polymer chains have consequently been used in a variety of optoelectronic devices such as organic field-effect transistors (OFET) [9], organic solar cell-integrated liquid crystal displays [5], or bipolarized light-emitting diodes [10]. In particular, epitaxial growth using crystallite templates formed by 1,3,5-trichlorobenzene (TCB) has shown remarkable results when applied to OFET with hole mobilities values 2.75 to 4.66 times higher than those observed in conventionally deposited devices [9]. However, these values are only obtained from local measurements with interelectrode spacing (channel width) of $80 \mu \mathrm{m}$. Deposition processes based on TCB can also be applied to the formation of films with polarized light-absorption properties [11], which could, for example, be used in analytical equipment for light-polarization analysis (e.g., cosmic rays). Using a universal method that can be used with a wide range of conjugated polymers independently of their crystalline behavior is consequently of major interest for the field of materials science. Furthermore, to be able to practically use thin films containing aligned conjugated polymer chains, the chain orientation should be achieved on a relatively large scale (areas of $1 \mathrm{~cm}^{2}$ or more). Here, we demonstrate that while techniques such as electrospinning and rubbing can give extremely positive results locally, they do not always 


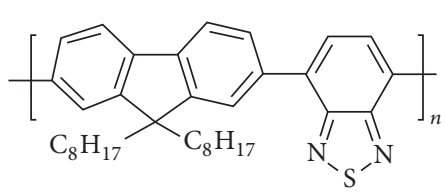

F8BT

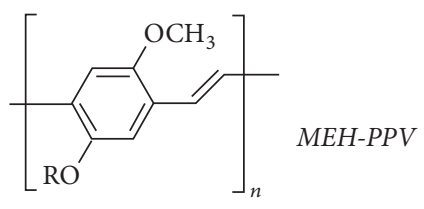

$\mathrm{R}=* \overbrace{\mathrm{CH}_{3}}^{\mathrm{CH}_{3}}$<smiles>CC(C)(C)CC(c1ccccc1)C(C)(C)C</smiles><smiles>Clc1ccccc1</smiles><smiles>Clc1cc(Cl)cc(Cl)c1</smiles>

Figure 1: Chemical structures and properties of materials used in this study.

match the requirements for large area fabrication. Consequently, we introduce a new method combining off-center spin-coating and epitaxial growth with TCB, which shows large increases in polarized emission from films obtained with conjugated polymers exhibiting various crystalline behaviors.

\section{Experimental Section}

2.1. Materials. Chlorobenzene (CB) and TCB, poly(9,9dioctylfluorene-alt-benzothiadiazole) (F8BT, Mn 17,00023,000), and polystyrene (PS, Mw 35,000) were purchased from Sigma-Aldrich and used as received. Poly[2-methoxy-5(2-ethylhexyloxy)-1,4-phenylenevinylene] (MEH-PPV) was obtained from 1-Material. The chemical structures of the materials used in this study are summarized in Figure 1.

\subsection{Nanofiber and Thin Film Formation. The electrospinning} process was performed using a F8BT:PS blend containing 10 to $25 \mathrm{wt} \%$ of F8BT with respect to the total polymer weight. The solutions in CB were optimized at a concentration of $200 \mathrm{mg} / \mathrm{ml}$ to obtain the adequate viscosity for the electrospinning process. A potential of $15 \mathrm{kV}$ was applied to the metal spinneret placed $10 \mathrm{~cm}$ from the target electrodes (Figure $2(\mathrm{a})$ ). A potential of $-5 \mathrm{kV}$ was applied to the two parallel target electrodes alternatively with a switching time of $10 \mathrm{~s}$ to fabricate aligned nanofibers in the space between the electrodes. An additional elongation/alignment step was applied to the fibers by increasing the space between the two electrodes from 2 to $3 \mathrm{~cm}$ after fiber deposition $[3,4]$. The fibers were then collected on a glass substrate cleaned by a standard cleaning procedure (ultrasonication in acetone and hot isopropanol).

Rubbing was performed using a nylon cloth (Savina ${ }^{\circledR} \mathrm{MX}$, purchased from KB Seiren) on F8BT films deposited from a $10 \mathrm{mg} / \mathrm{ml}$ solution in CB by spin-coating on cleaned glass at $1000 \mathrm{rpm}$ for $60 \mathrm{~s}$ (Figure 2(b)). The applied pressure and number of repetitions for the rubbing process were optimized to $500 \mathrm{kPa}$ and 10 times, respectively. Note that, at lower applied pressure and rubbing times, no polarized emission could be observed.

All spin-coated films were deposited using $10 \mathrm{mg} / \mathrm{ml}$ conjugated polymer solutions in $\mathrm{CB}$ at $1000 \mathrm{rpm}$ for $60 \mathrm{~s}$. An additional amount of TCB $(200 \mathrm{mg} / \mathrm{ml})$ was added to the solutions for epitaxial growth using crystallite templates. Off-center spin-coating was performed by depositing the solution $1 \mathrm{~cm}$ away from the center of the spin-coating stage (Figure $2(\mathrm{c})$ ). Note that only small variations in polarization ratios $\left(P_{R}\right)$ were observed when the solution was deposited $2 \mathrm{~cm}$ away from the center of the spin-coating stage.

2.3. Nanofiber and Thin Film Characterizations. The polarized photoluminescence (PL) spectra were collected by exciting the samples with a LED with an emission peak at $410 \mathrm{~nm}$. A polarizer was placed between the samples and spectrometer (BLUE-Wave, StellarNet) and the data was collected with an integration time of $1 \mathrm{~s}$ averaged over 5 measurements for each polarization angle (every 15 degrees from 0 to 180 degrees). Note that no major differences could be observed between the 0 and 180 degrees measurements. This confirms that no photodegradation of the conjugated polymer occurred during the PL characterization under these mild excitation conditions. $P_{R}$ was calculated by dividing the integration of the highest intensity PL peak by the lowest one (polarizer placed at 90 degrees with respect to the highest intensity).

Optical and atomic force microscopy (AFM) images were performed using bright-field and contact mode, respectively. Fluorescence and bright-field optical images were obtained using Nikon Eclipse and Olympus DSX510 microscopes, respectively. X-ray diffraction (XRD) patterns carried out in Bragg-Brentano geometry were obtained at $25^{\circ} \mathrm{C}$ using a Siemens D500 diffractometer equipped with a sensible detector (VORTEX), Soller slits $\left(2^{\circ}\right)$, and narrow slits $\left(0.3^{\circ}\right)$ and a Siemens FK 60-10 2000 W tube $\left(\mathrm{Cu} \mathrm{K}_{\alpha}\right.$ radiation, $\lambda=$ $0.154 \mathrm{~nm})$. The operating voltage and current were $45 \mathrm{kV}$ and 


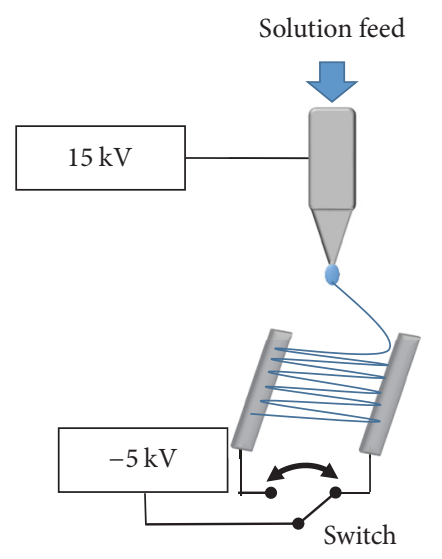

(a) Electrospinning setup

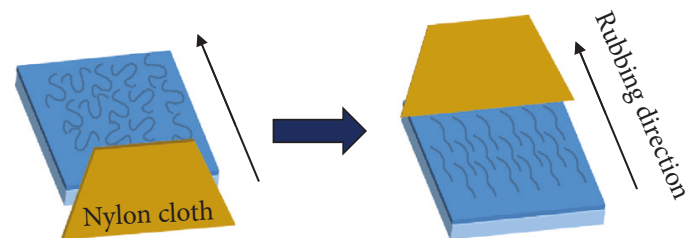

(b) Rubbing

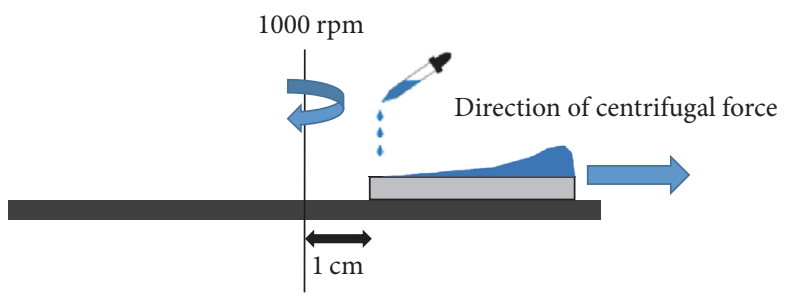

(c) Off-center spin-coating

FIGURE 2: Schematic representations of (a) the electrospinning, (b) the rubbing, and (c) the off-center spin-coating processes.

$40 \mathrm{~mA}$, respectively. Data were collected from $3^{\circ}$ to $30^{\circ}(2 \theta)$ at $0.05^{\circ}$ intervals ( $9 \mathrm{~s}$ for each one).

\section{Results and Discussion}

3.1. Locally Polarized Emission in Electrospun Fibers and Rubbed Films. As mentioned in Introduction, to generate aligned conjugated thin films for practical use involving polarized light, the polarized emission properties should extend to large areas. Electrospinning of conjugated polymers has been extensively studied to obtain conjugated polymer alignment and/or to observe electroluminescence from these fibers [12]. Here we should emphasize the fact that as conjugated polymers have a rigid backbone, electrospinning of pure conjugated polymers can hardly be done and they are commonly blended with other stretchable polymer materials to obtain the adequate viscoelastic properties for the electrospinning process $[3,4,12]$. Consequently, we adapted a standard recipe and combined it with our electrospinning setup for aligned electrospun fiber preparation. The number of collected fibers is directly proportional to the number of switching times which gave us the opportunity to observe the PL behaviors of single fiber (Figure 3(a)) and aligned fiber bundles (Figure 3(b)).

While electrospinning on conventional collector screens (round shaped aluminum counter electrodes) gave relatively smooth fibers, using the alignment setup increased the density of bead formation during the deposition/elongation processes. Strong polarization could be observed from the stretched F8BT:PS single fibers in the nonbeaded areas (Figure 3(a)). However, Figure 3(a) also emphasizes that unpolarized and strong fluorescence is emitted from the beads. Figure 3(b) clearly indicates that aligned fiber bundles can be fabricated using our electrospinning setup. Nonetheless, the large amount of unpolarized beads has a much larger $\mathrm{PL}$ intensity as compared to the thin smooth fibers and, consequently, no polarization could be observed from these samples $\left(P_{R} \sim 1\right)$.

Similar issues arise in the case of rubbed samples. While the rubbed F8BT films display relatively high polarization in the smooth parts, the rubbing process itself generates some bead-like large aggregates which do not display any polarized emission properties (Figure 3(c)). The images in Figure 3(c) contain unrubbed and rubbed parts of the same sample deposited on a $1 \mathrm{~cm}^{2}$ substrate. The image corresponds to the part of the sample (approx. $0.15 \times 0.25 \mathrm{~cm}^{2}$ ) containing the lowest amount of rubbing-induced beads and scratches. The two images obtained using perpendicular polarizer orientations suggest that a strong $P_{R}$ should be expected from these samples. The polarized PL measurements (Figure 3(d)) contradict these assumptions due to the large number of unpolarized beads in the measured areas $\left(1 \mathrm{~cm}^{2}\right)$. The highest $P_{R}$ value for rubbed films $\left(P_{R}=1.25\right)$ was obtained by applying a rubbing pressure of $500 \mathrm{kPa}$ and after 5 rubbings. Note that higher values can be obtained when using other conjugated polymers with larger crystallinity such as poly(3hexylthiophene) upon rubbing in similar conditions. Additionally, polarized emission from F8BT has been demonstrated when using rubbed polyimide templates on which F8BT is deposited but the formation of beads upon direct rubbing of this small crystallinity polymer may explain why a multistep process (rubbing the polyimide template followed 


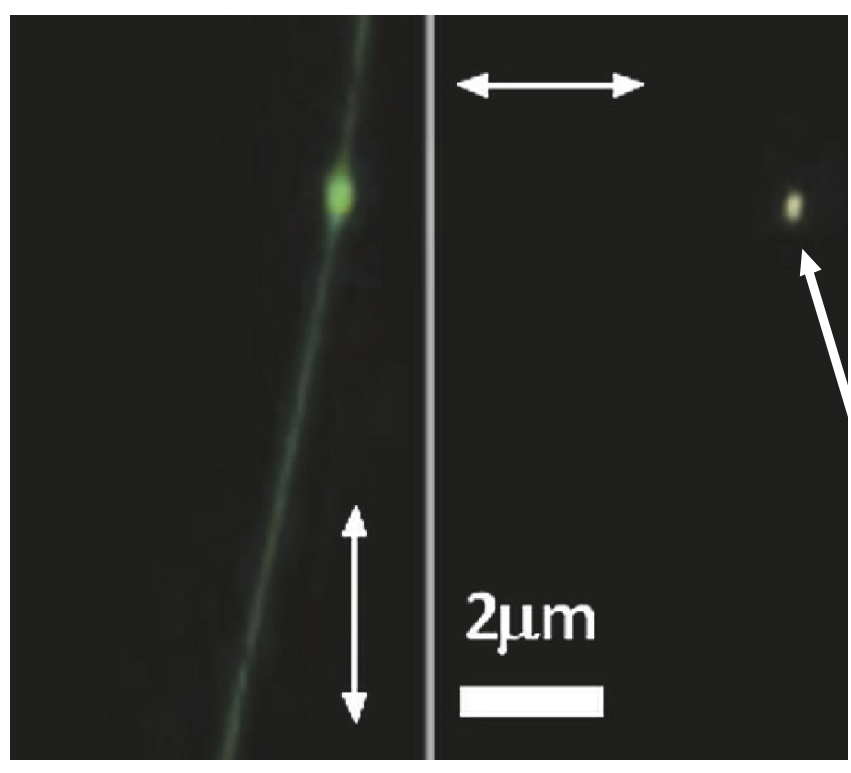

(a)

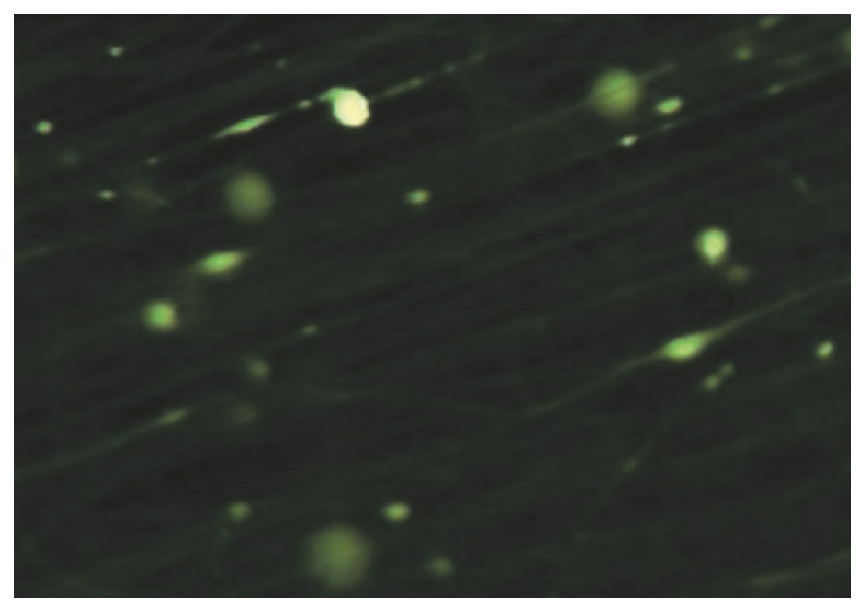

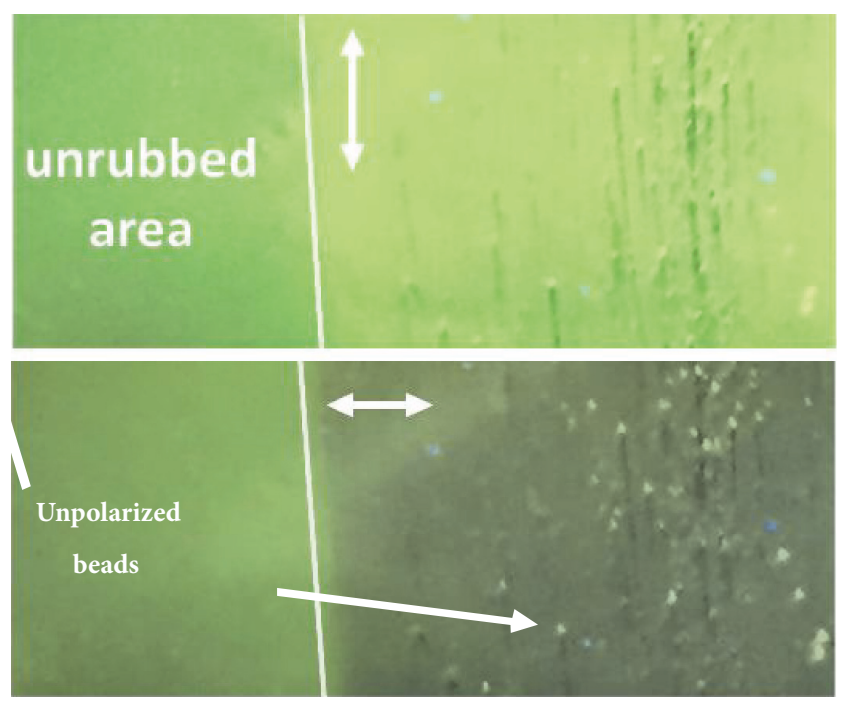

(c)

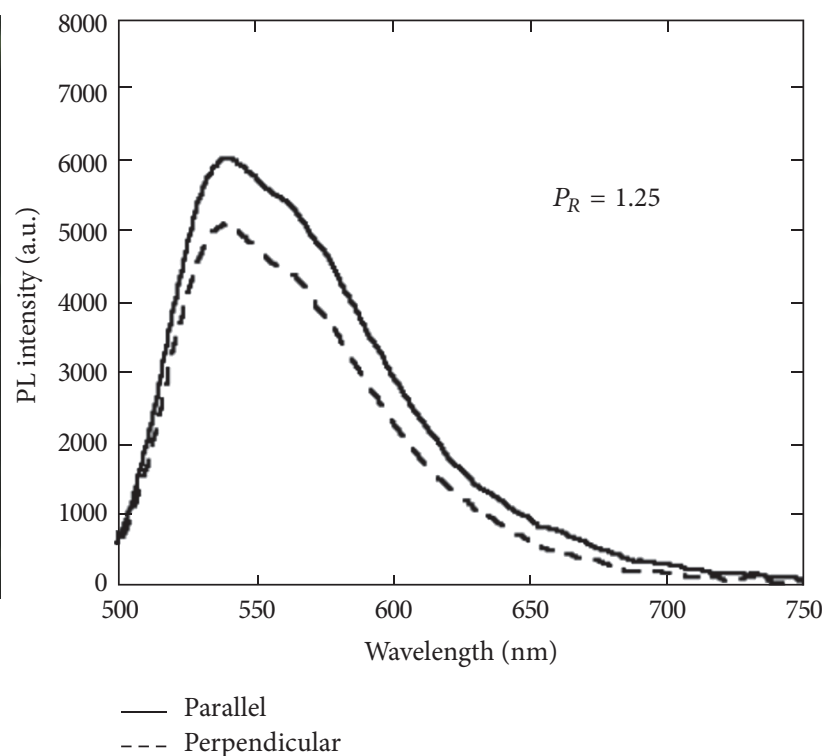

(d)

Figure 3: Fluorescence microscopy images of (a) a single and (b) a bundle of F8BT:PS electrospun fibers. (c) Fluorescence photograph and (d) PL spectra of rubbed F8BT films. The two-side arrows represent the direction of the polarizer placed between the sample and camera.

by deposition of F8BT) is necessary $[13,14]$. Nonetheless, the scope of this study is to introduce a universal process to generate polarized conjugated polymer thin films and, consequently, in the next section, we propose a method that provides high $P_{R}$ even for small crystallinity materials such as F8BT.

3.2. Ordering of Conjugated Polymer Films by Epitaxial Growth during Spin-Coating. Epitaxial growth during spincoating using TCB has been previously studied using a diketopyrrolopyrrole-based conjugated polymer to increase the charge transport properties of the polymer used in $\mathrm{p}$ type OFET [9]. The material used displayed strong crystalline peaks without the addition of TCB which, in this case, only induces radial growth and the formation of large spherulites. Here, we discuss and demonstrate that similar formation of large spherulites composed of oriented nanofibers can be obtained when using conjugated polymers with smaller crystallinity such as F8BT (Figure 4(a)). TCB crystallites are formed during spin-coating in a radial direction and are then used as templates or nucleation centers that interact with the conjugated polymer molecules (Figure 4(b)). 


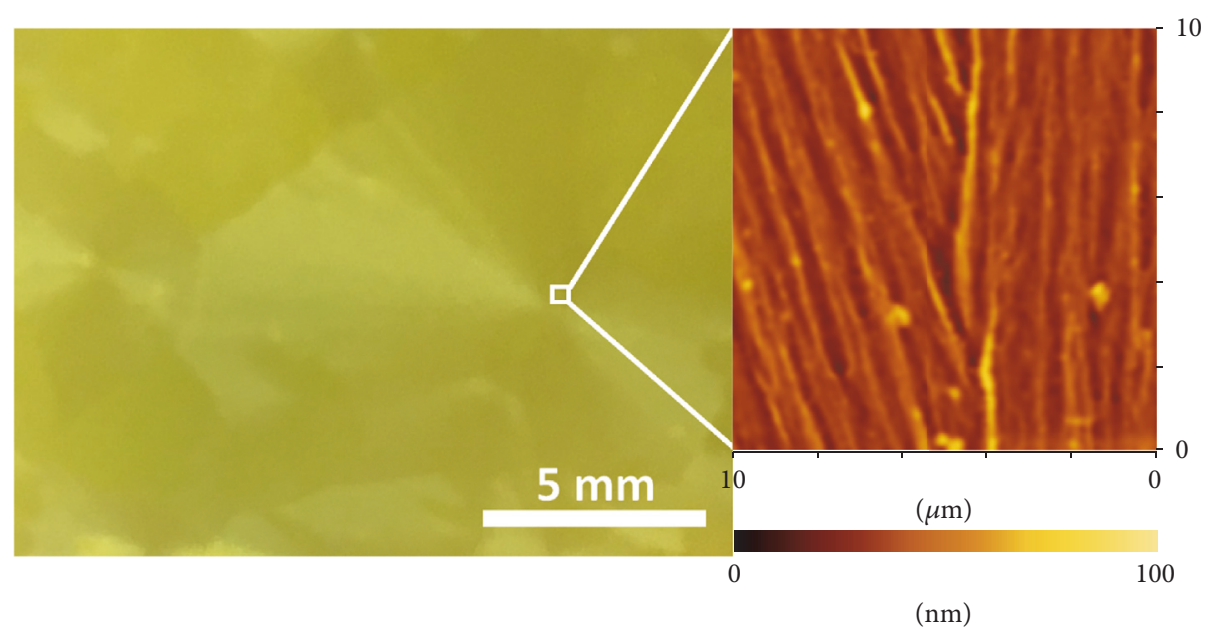

(a)

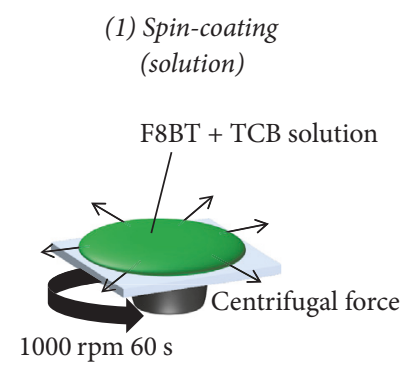

(2) TCB condensation (wet film)

TCB crystallite template

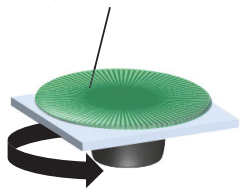

(b)
(3) F8BT fiber formation (dried film)

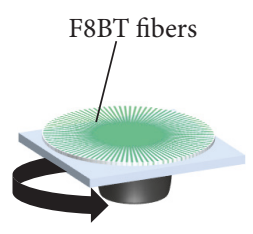

FIGURE 4: (a) Morphological characterization (photograph and AFM) and (b) schematic representation of the mechanism of F8BT radial nanofiber formation.

While the formation of spherulites and nanofibers can be clearly observed by eye in both F8BT and MEH-PPV samples, it is important to understand to which degree these structures are ordered and whether the addition of TCB to the spin-coated solution can force the crystallization of the two polymer materials. In as-spun films of F8BT or MEH-PPV, the degree of order achieved is insufficient to assess a real detectable effect by XRD (Figure 5) [15-17]. As previously demonstrated, a higher degree of order may be obtained by annealing these films at high temperature [17]. Although the inclusion of TCB into the conjugated polymer solution leads to the formation of fiber-like structures, no interchain crystallinity peak could be measured at the center of films spin-coated from solutions containing TCB. In fact, XRD patterns at the center of these films are similar to those from reference samples fabricated without TCB (data not shown). Interestingly, when measuring areas approximately $0.5 \mathrm{~cm}$ away from the center of the substrate, XRD peaks typically ascribed to interchain molecular packing can be observed in both F8BT and MEHPPV samples prepared using TCB. These peaks (marked with arrows in Figure 5) correspond to $d$-spacing (interchain spacing) of 1.7 and $1.85 \mathrm{~nm}$, respectively, for F8BT and MEHPPV.
3.3. Directional Epitaxial Growth Using Off-Center SpinCoating. Epitaxial growth resulting from the inclusion of TCB in the spin-coated solution leads to the formation of highly ordered nanofiber structures that are relatively aligned when observing the samples away from the center of the spherulites. Samples prepared from solutions containing TCB concentrations of $250 \mathrm{mg} / \mathrm{ml}$ lead to the formation of large spherulites that cover areas larger than $1 \mathrm{~cm}^{2}$. However, as the fibers grow in a radial manner away from the center, the samples do not display any clear polarization and various $P_{R}$ can be measured depending on the measurement position relative to the center of the sample. Here, we explore a simple method for directional deposition, namely, offcenter spin-coating, and the morphology and polarization properties of films formed by off-center spin-coating of polymer solutions containing TCB. XRD patterns from F8BT and MEH-PPV samples prepared using off-center epitaxial growth are presented in Figure 5. Note that no major difference in XRD patterns was observed for the edge of center spin-coating samples (mentioned in Section 3.2) and off-center samples containing TCB. Additionally, similar thin film properties could be found when the off-center spincoating was performed at distances of 1 and $2 \mathrm{~cm}$ from the center of the spin-coating stage. The results presented here 


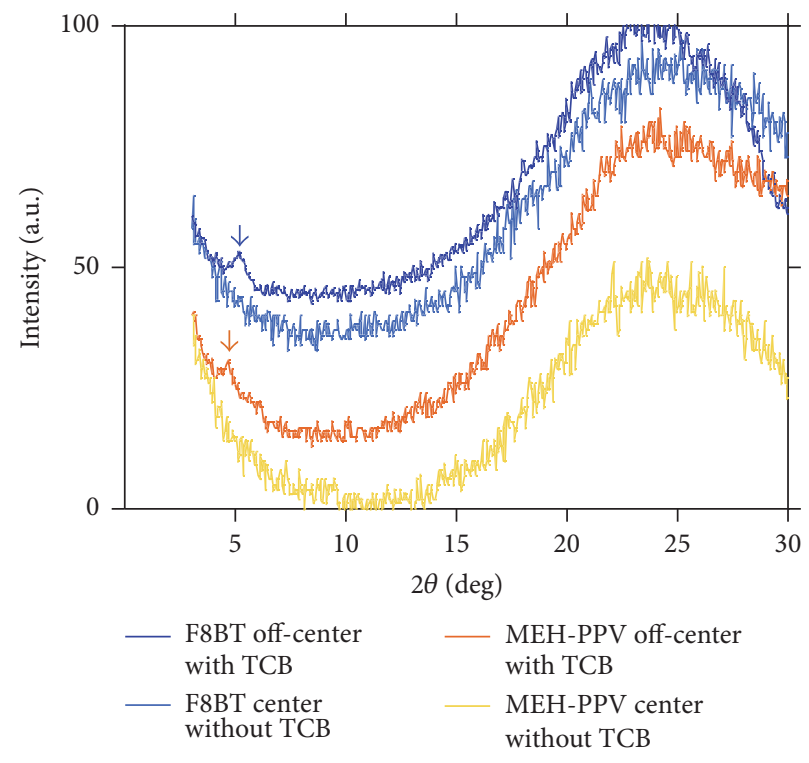

FIGURE 5: F8BT and MEH-PPV XRD pattern observed either at the center of spin-coated samples without TCB or in off-center spin-coated samples with TCB. The dark blue and orange arrows correspond to the molecular packing peaks for F8BT (at 5.1 ${ }^{\circ}, 1.7 \mathrm{~nm}$ ) and MEH-PPV (at $\left.4.8^{\circ}, 1.85 \mathrm{~nm}\right)$, respectively.

correspond tovspace.5pt those obtained when the edge of the substrate was placed $1 \mathrm{~cm}$ away from the center of the spincoating stage (Figure 6). The bright-field optical microscopy images clearly emphasize that the quality of the fibers and their alignment increases with distance from the spin-coating center. In fact, in the central part of the conventional spincoated samples, multiple growth directions (three in the image presented in Figure 6) can be found. Furthermore, both the optical micrograph and the AFM image in Figure 4 suggest that only small size fibers are formed close to the center, which are mixed with conjugated polymer aggregates. On the other hand, the edge of the samples deposited using conventional spin-coating with TCB already displays structures with a higher degree of orientation, which is further improved when using off-center spin-coating. The optical micrographs presented in Figure 6 correspond to samples obtained using F8BT but a similar trend can be observed for MEH-PPV samples. The major morphological difference between F8BT and MEH-PPV fibers resides in their dimensions. MEH-PPV fibers are thicker and have a smaller height as compared to equivalent F8BT fibers formed at similar distances from the spin-coating center. Nonetheless, these results correlate well with the XRD patterns shown in Figure 5 as we can clearly observe that fine fibers with enhanced crystallinity are formed in off-center spin-coated samples prepared from solutions containing TCB.

The fiber-like structure formation observed in our samples (Figure 6) only occurs with the addition of TCB molecules into the polymer solutions. In fact, the formation of large spherulite structures during center spin-coating strongly suggests that the TCB molecule crystallization is formed along a preferential direction which corresponds to the solution spreading/drying direction in which the centrifugal forces are applied. As these templates induce the conjugated polymer fiber formation, controlling the spreading direction of the solution is the key parameter to form aligned fiber-like structures. This could be achieved by increasing the strength of the applied centrifugal force using off-center spin-coating.

The enhanced fiber alignment observed in the optical micrographs in Figure 6 can be easily understood when taking into account the intensity of the centrifugal forces applied to the sample under each condition. In fact, the intensity of the centrifugal force $\left(F_{c}\right)$ applied to the solution can be expressed as $F_{c}=m \omega^{2} r$, where $m, \omega$, and $r$ correspond to the mass, angular speed, and radius, respectively. As $\omega$ is kept constant at $1000 \mathrm{rpm}$ as well as the volume of deposited solution for each sample, the only parameter that varies in the above-mentioned equation is $r$. Consequently, we can approximate that $F_{c}$ is 5 and 10 to 20 times stronger at the edge of the conventional spin-coated samples and in the offcenter spin-coated samples relatively to the central part of conventional spin-coated samples, respectively. Furthermore, we verified that orientation of the fibers and the conjugated molecules was actually obtained by measuring the polarized PL spectra of all samples and calculating their $P_{R}$ (Figure 7). Note that no polarized emission properties could be observed for samples without TCB measured on different areas of center and off-center spin-coated substrates. 


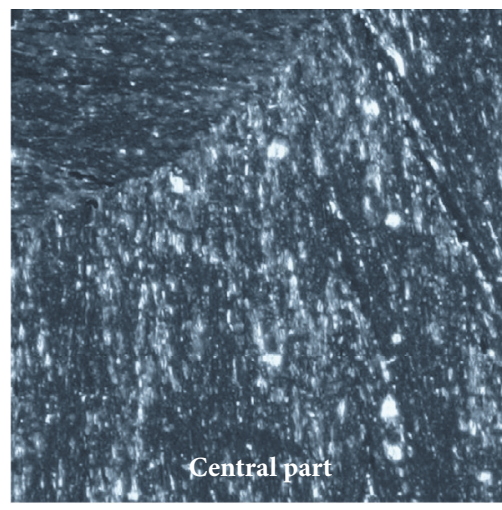

Edge $(0.5-0.6 \mathrm{~cm}$ from center $)$

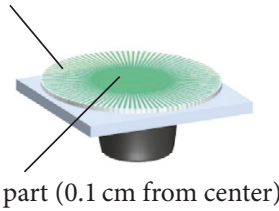

Central part $(0.1 \mathrm{~cm}$ from center $)$

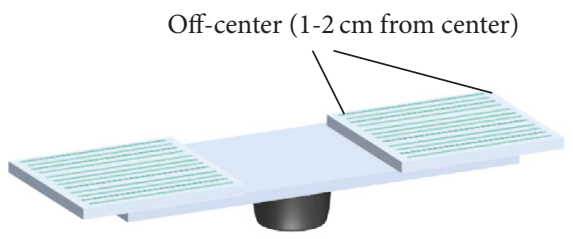

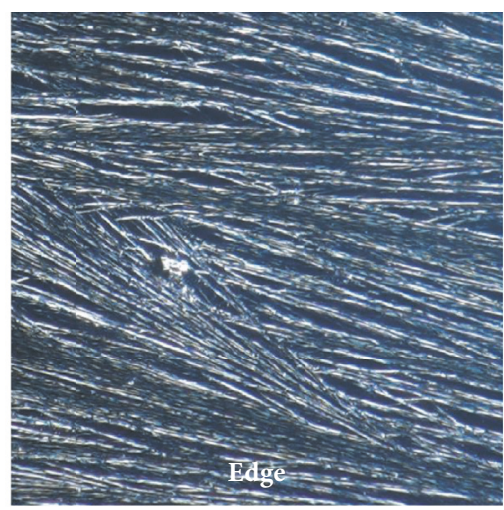

F8BT

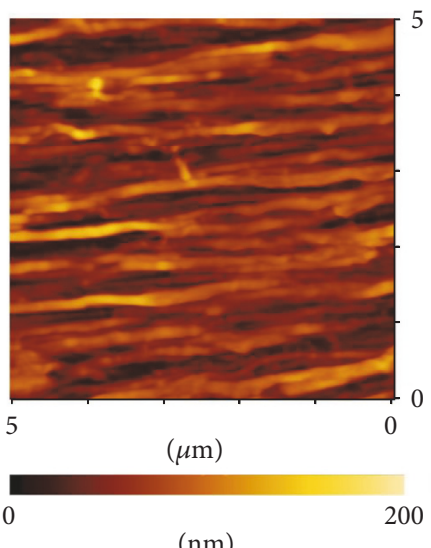

$(\mathrm{nm})$

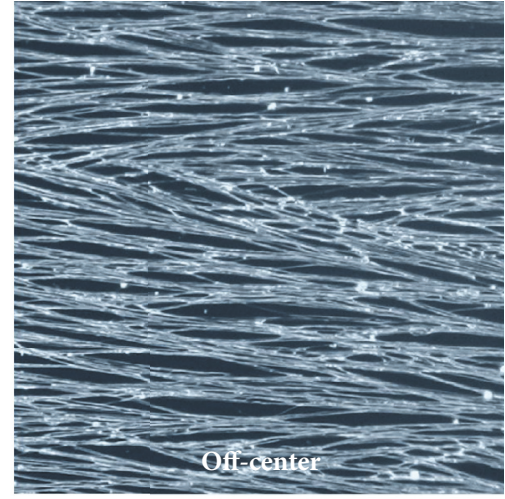

$M E H-P P V$

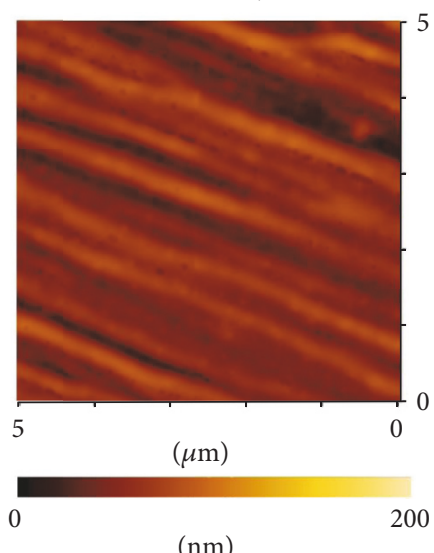

FIGURE 6: Optical micrographs $\left(200 \times 200 \mu \mathrm{m}^{2}\right)$ of conventional (central part, edge) and off-center spin-coated (off-center) films produced from F8BT solutions containing TCB. AFM images of nanofibers formed using off-center spin-coating from F8BT and MEH-PPV solutions containing TCB.

The polarized PL spectra in Figure 7 clearly demonstrate that while no polarized emission can be observed in samples without TCB, the addition of TCB and the increasing distance from the spin-coating center result in large enhancements of $P_{R}$. The F8BT samples prepared using TCB display $P_{R}$ values of 1.35 and 2.24 for center (edge) and off-center spincoating, respectively. The corresponding values for $\mathrm{MEH}-$ PPV are even higher and reach 2.84 and 4.09 for center and off-center spin-coated samples, respectively. Furthermore, it may be worth remarking that the PL spectra of samples containing TCB display more structured spectral shapes with pronounced shoulders at 580 and $640 \mathrm{~nm}$, respectively, forF8BT and MEH-PPV. Once again, this phenomenon agrees well with the crystallinity enhancement observed by XRD (Figure 5) as these enhanced shoulders are typically ascribed to an increase in interchain interactions of the conjugated polymer materials [18]. The highest polarized PL intensities for off-center spin-coated samples with TCB are measured at angles of 15 and 30 degrees with respect to the fiber axis for F8BT and MEH-PPV, respectively. This deviation with respect to the nanofiber axis suggests that the conjugated polymer chains are not perfectly aligned inside the fiber. However, one should also take into account the fact that the optical transition dipole moments of the conjugated polymers are not always parallel to their backbone axis [19]. In general, higher values of $P_{R}$ are obtained for MEH-PPV. This is most probably resulting from the fact that, unlike F8BT which is composed of two different monomers, the transition dipole moment of MEH-PPV has a higher probability of being parallel to its planar conjugated backbone.

\section{Conclusions}

In summary, we have demonstrated a universal method to generate thin films of conjugated polymers composed of aligned nanofiber-like structures. This was achieved by combining two well-known methods for polymer chain alignment, namely, epitaxial growth using TCB and offcenter spin-coating. Our results further emphasize the fact that these two methods cannot generate large-scale and high polarization ratio films if used independently. The thin films displaying the highest polarization reach $P_{R}$ values of approximately 4.1. As this approach can be applied to virtually any conjugated polymer material, the process we introduce here is very promising when it comes to fabricating new polarized light-emitting-based technologies. 

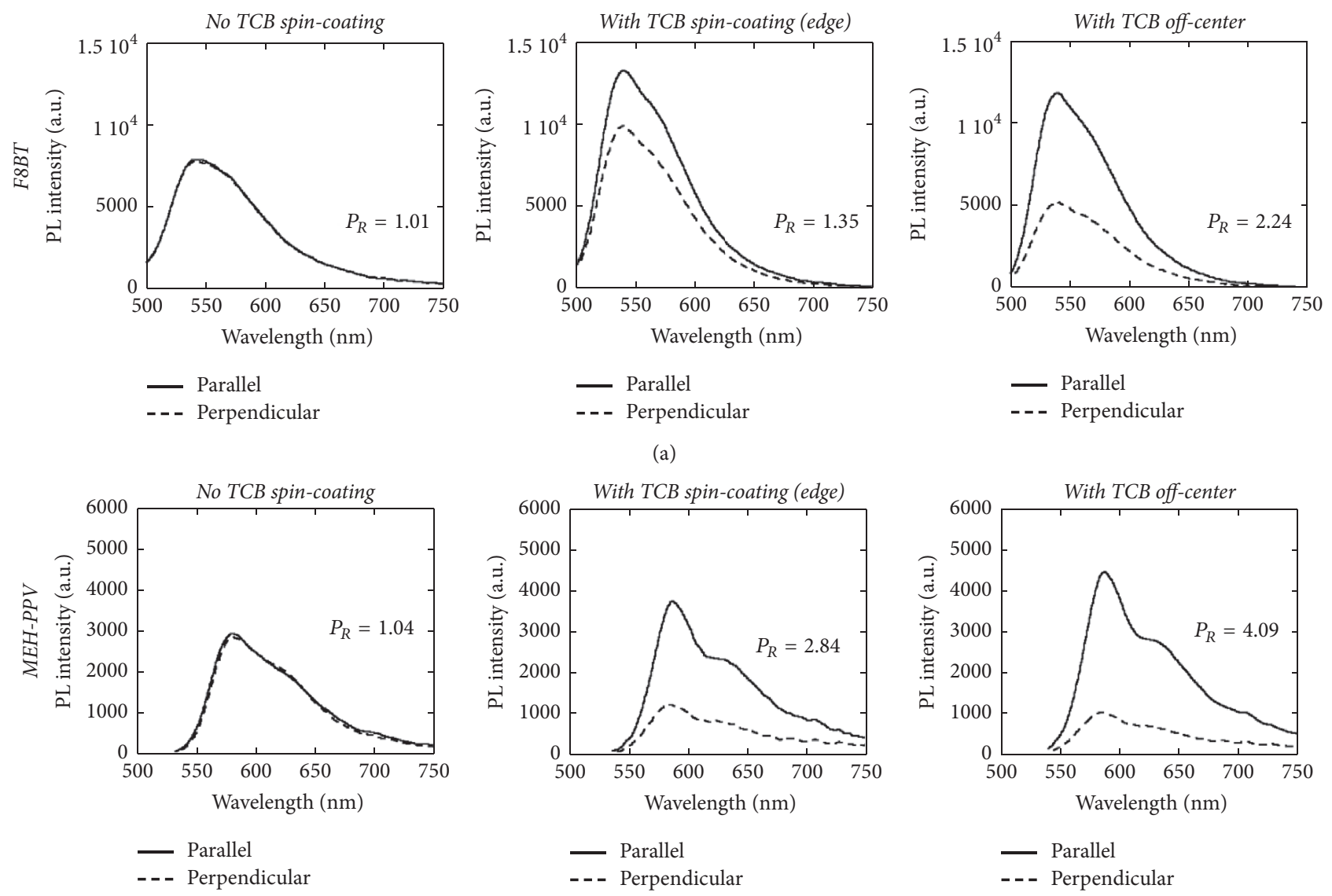

Figure 7: Polarized PL spectra of (a) F8BT and (b) MEH-PPV samples prepared using center spin-coating with no TCB or with TCB at the edge of center spin-coated samples and from off-center samples.

\section{Conflicts of Interest}

The authors declare that they have no conflicts of interest.

\section{Acknowledgments}

This work was supported by the University of ElectroCommunications Financial Support for Young Faculty Members.

\section{References}

[1] J. H. Burroughes, D. D. C. Bradley, A. R. Brown et al., "Lightemitting diodes based on conjugated polymers," Nature, vol. 347, no. 6299, pp. 539-541, 1990.

[2] V. Vohra and T. Anzai, "Molecular orientation of conjugated polymer chains in nanostructures and thin films: Review of processes and application to optoelectronics," Journal of Nanomaterials, vol. 2017, Article ID 3624750, 18 pages, 2017.

[3] Y. Ishii, H. Sakai, and H. Murata, "A new electrospinning method to control the number and a diameter of uniaxially aligned polymer fibers," Materials Letters, vol. 62, no. 19, pp. 3370-3372, 2008.
[4] M. Campoy-Quiles, Y. Ishii, H. Sakai, and H. Murata, "Highly polarized luminescence from aligned conjugated polymer electrospun nanofibers," Applied Physics Letters, vol. 92, no. 21, Article ID 213305, 2008.

[5] R. Zhu, A. Kumar, and Y. Yang, "Polarizing organic photovoltaics," Advanced Materials, vol. 23, no. 36, pp. 4193-4198, 2011.

[6] V. Vohra, G. Arrighetti, L. Barba, K. Higashimine, W. Porzio, and H. Murata, "Enhanced vertical concentration gradient in rubbed P3HT:PCBM graded bilayer solar cells," The Journal of Physical Chemistry Letters, vol. 3, no. 13, pp. 1820-1823, 2012.

[7] Y. Yuan, G. Giri, A. L. Ayzner et al., "Ultra-high mobility transparent organic thin film transistors grown by an off-centre spin-coating method," Nature Communications, vol. 5, article 3005,2014

[8] C. Müller, M. Aghamohammadi, S. Himmelberger et al., "Onestep macroscopic alignment of conjugated polymer systems by epitaxial crystallization during spin-coating," Advanced Functional Materials, vol. 23, no. 19, pp. 2368-2377, 2013.

[9] J. Y. Kim, D. S. Yang, J. Shin et al., "High-performing thin-film transistors in large spherulites of conjugated polymer formed by epitaxial growth on removable organic crystalline templates," ACS Applied Materials \& Interfaces, vol. 7, no. 24, pp. 1343113439, 2015. 
[10] A. Bolognesi, C. Botta, D. Facchinetti et al., "Polarized electroluminescence in double-layer light-emitting diodes with perpendicularly oriented polymers," Advanced Materials, vol. 13, no. 14, pp. 1072-1075, 2001.

[11] B. Dorling, V. Vohra, T. T. Dao, M. Garriga, H. Murata, and M. Campoy-Quiles, "Uniaxial macroscopic alignment of conjugated polymer systems by directional crystallization during blade coating," Journal of Materials Chemistry C, vol. 2, no. 17, pp. 3303-3310, 2014.

[12] V. Vohra, U. Giovanella, R. Tubino, H. Murata, and C. Botta, "Electroluminescence from conjugated polymer electrospun nanofibers in solution processable organic light-emitting diodes," ACS Nano, vol. 5, no. 7, pp. 5572-5578, 2011.

[13] D.-M. Lee, Y.-J. Lee, J.-H. Kim, and C.-J. Yu, "Birefringencedependent linearly-polarized emission in a liquid crystalline organic light emitting polymer," Optics Express, vol. 25, no. 4, pp. 3737-3742, 2017.

[14] S. I. Jo, Y. Kim, J.-H. Baek, C.-J. Yu, and J.-H. Kim, "Highly polarized emission of the liquid crystalline conjugated polymer by controlling the surface anchoring energy," Japanese Journal of Applied Physics, vol. 53, no. 3, Article ID 03CD04, 2014.

[15] V. Vohra, W. Mróz, S. Inaba, W. Porzio, U. Giovanella, and F. Galeotti, "Low-cost and green fabrication of polymer electronic devices by push-coating of the polymer active layers," ACS Applied Materials \& Interfaces, vol. 9, no. 30, pp. 25434-25444, 2017.

[16] R. J. Kline, M. D. McGehee, and J. Macromol, "Morphology and charge transport in conjugated polymers," Journal of Macromolecular Science, Part C: Polymer Reviews, vol. 46, no. 1, pp. 27-45, 2006.

[17] S. H. Chen, A. C. Su, H. L. Chou, K. Y. Peng, and S. A. Chen, "Phase behavior and molecular aggregation in bulk poly(2methoxy-5-(2I-ethylhexyloxy)-1,4-phenylenevinylene)," Macromolecules, vol. 37, no. 1, pp. 167-173, 2004.

[18] O. Y. Posudievsky, M. S. Papakin, O. P. Boiko, V. G. Koshechko, and V. D. Pokhodenko, "Enhanced and tunable photoluminescence of polyphenylenevinylenes confined in nanocomposite films," Nanoscale Research Letters, vol. 10, no. 1, pp. 1-7, 2015.

[19] T. W. Hagler, "Nonparallel transition dipole moments and the polarization dependence of electroabsorption in nonoriented conjugated polymer films," Chemical Physics Letters, vol. 218, no. 3, pp. 195-199, 1994. 

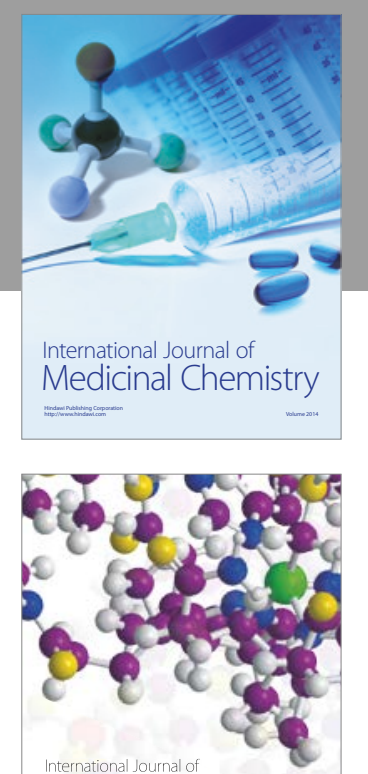

Carbohydrate Chemistry

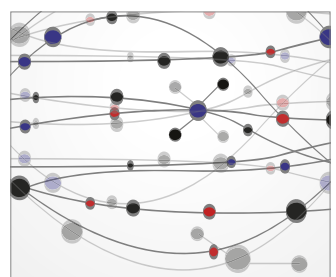

The Scientific World Journal
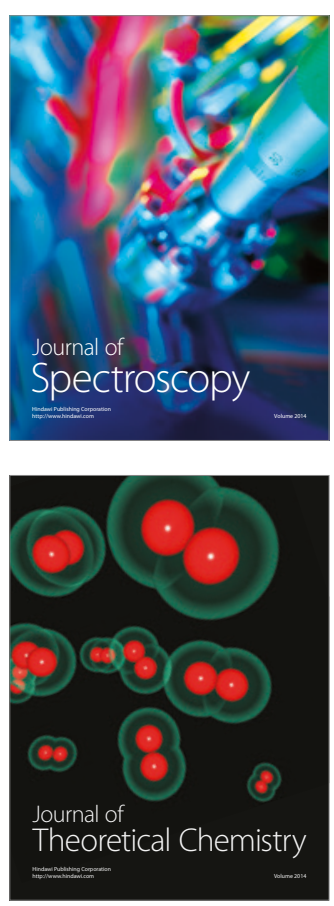
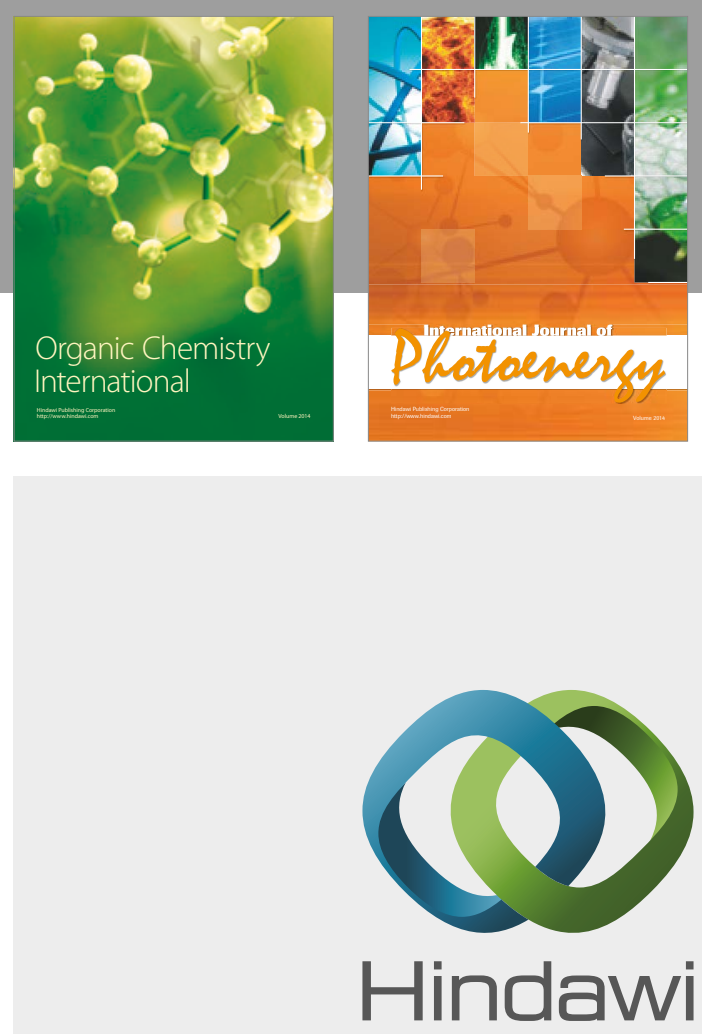

Submit your manuscripts at

https://www.hindawi.com

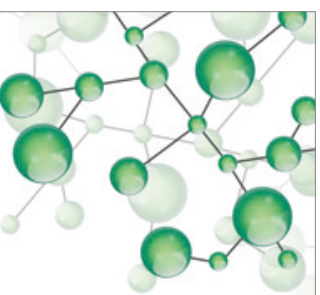

International Journal of

Inorganic Chemistry

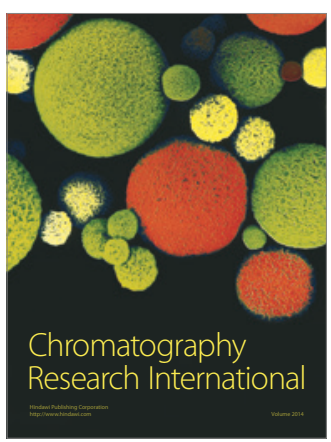

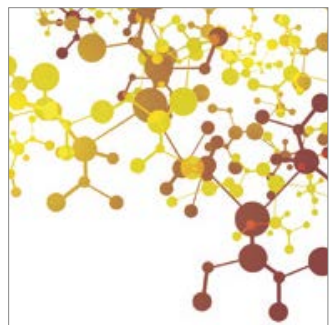

Applied Chemistry
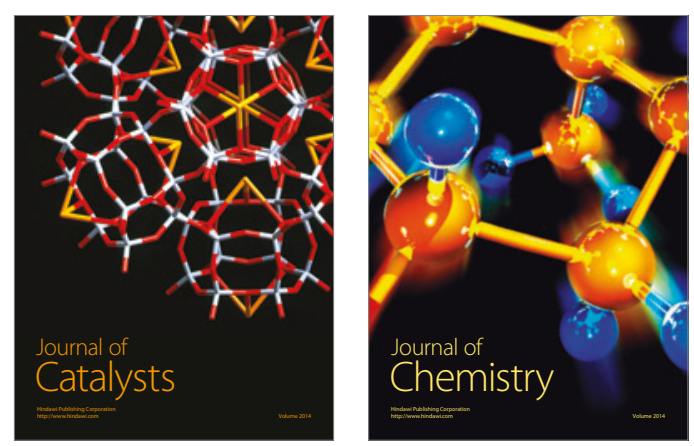
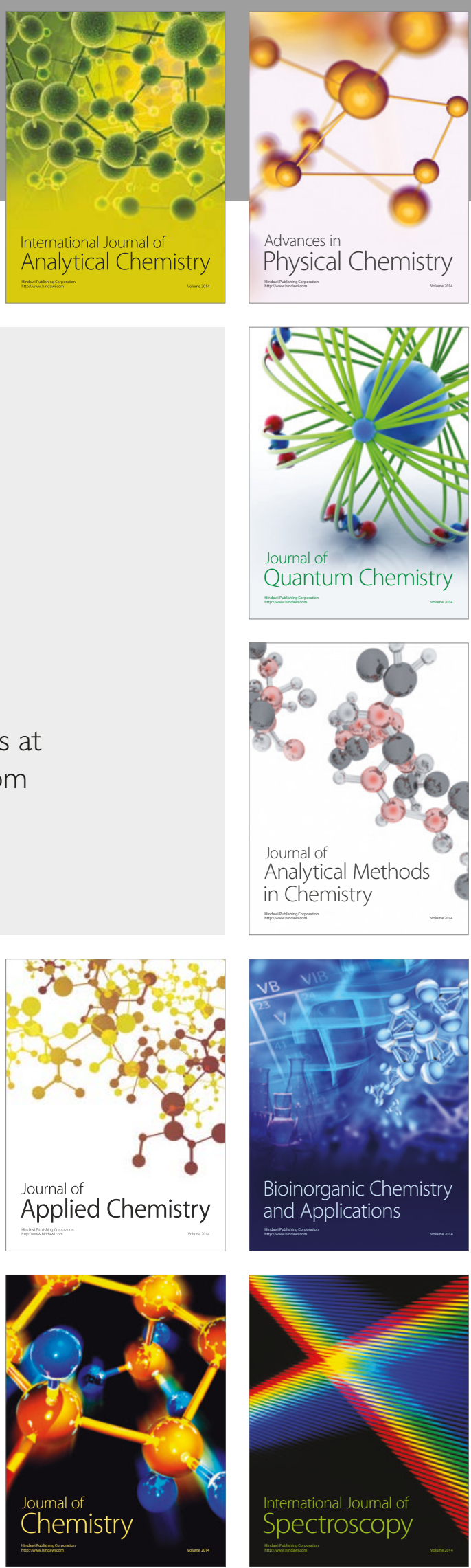